Revista de Matemática: Teoría y Aplicaciones 2006 13(1) : 35-39

CIMPA - UCR - CCSS ISSN: 1409-2433

\title{
A FORMULA FOR COMPLEX ZONAL POLYNOMIALS OF SECOND ORDER
}

\author{
Francisco J. Caro-Lopera * José A. Díaz-García ${ }^{\dagger}$ \\ GraCIELA GONZÁLEZ-FARÍAs ${ }^{\ddagger}$
}

Recibido/Received: 22 Nov 2005 - Aceptado/Accepted: 30 Ago 2006

\begin{abstract}
A formula for complex zonal polynomials of second order is derived by solving a particular partial differential equation.
\end{abstract}

Keywords: Laplace-Beltrami operator, zonal polynomials, Hermitian matrix, Legendre's differential equation.

\section{Resumen}

En el presente trabajo se encuentra una fórmula para polinomios zonales complejos de segundo orden, a partir de una ecuación diferencial parcial.

Palabras clave: Operador de Laplace-Beltrami, polinomios zonales, matriz hermitiana, ecuación diferencial de Legendre.

Mathematics Subject Classification: 62H05, 33A70.

\section{Introduction}

Recently Díaz-García and Caro-Lopera (2006b) computed the zonal polynomials of positive definite hermitian matrix by the use of the Laplace-Beltrami operator. In the same

${ }^{*}$ Department of Basic Mathematics, Centro de Investigación en Matemáticas, Callejón de Jalisco s/n, Mineral de Valenciana, 36240 Guanajuato, Guanajuato, México. E-mail: fjcaro@cimat.mx

${ }^{\dagger}$ Department of Statistics and Computation, Universidad Autónoma Agraria Antonio Narro, 25315 Buenavista, Saltillo, Coahuila, México, E-Mail: jadiaz@uaaan.mx

${ }^{\ddagger}$ Department of Probability and Statistics, Centro de Investigación en Matemáticas, Callejón de Jalisco s/n, Mineral de Valenciana, 36240 Guanajuato, Guanajuato, México, E-Mail: farias@cimat.mx 
way for the real case, the zonal polynomials of positive definite and a semi-definite positive symmetric matrices were calculated by James (1968) and Díaz-García and Caro-Lopera (2006a), respectively. It is known that general formulae for complex and real zonal polynomials are not available, and the general partial differential equations for both polynomials are also unsolved. However, the differential equation for the zonal polynomials of definite positive symmetric matrix argument of the second order was solved by James (1968).

In this paper we reduce the partial differential equation for zonal polynomials of positive definite hermitian matrix of second order to a Hypergeometric differential equation type, which is analogous to the results obtained by James (1968) in the real case. By solving the ordinary differential equation, we get an explicit formula for the corresponding zonal polynomials, see Section 2.

\section{Complex Zonal Polynomials of Second Order.}

Let the partition $\kappa=\left(k_{1}, \ldots, k_{m}\right)$ of $k$ a decreasing sequence of nonnegative integers. In Díaz-García and Caro-Lopera (2006b) was proved that the zonal polynomials $\tilde{C}_{\kappa}(Y)$ of an $m \times m$ positive definite hermitian matrix $Y$ satisfy the partial differential equation

$$
\begin{aligned}
& \sum_{i=1}^{m} y_{i}^{2} \frac{\partial^{2}}{\partial y_{i}^{2}} \tilde{C}_{\kappa}(Y)+2 \sum_{i=1}^{m} \sum_{j=1(j \neq i)}^{m} y_{i}^{2}\left(y_{i}-y_{j}\right)^{-1} \frac{\partial}{\partial y_{i}} \tilde{C}_{\kappa}(Y)= \\
& {\left[\tilde{\rho}_{\kappa}+k(2 m-1)\right] \tilde{C}_{\kappa}(Y), }
\end{aligned}
$$

where

$$
\tilde{\rho}_{\kappa}=\sum_{i=1}^{m} k_{i}\left(k_{i}-2 i\right),
$$

$y_{1}, \ldots, y_{m}$ are the eigenvalues of the matrix $Y$ and $\kappa=\left(k_{1}, \ldots, k_{m}\right)$ is a partition of $k$.

When $m=2$ in (1) we get the partial differential equation

$$
\begin{aligned}
y_{1}^{2} \frac{\partial^{2} \tilde{C}}{\partial y_{1}^{2}}+y_{2}^{2} \frac{\partial^{2} \tilde{C}}{\partial y_{2}^{2}}+2 y_{1}^{2}\left(y_{1}-y_{2}\right)^{-1} \frac{\partial \tilde{C}}{\partial y_{1}}-2 y_{2}^{2}\left(y_{1}-y_{2}\right)^{-1} \frac{\partial \tilde{C}}{\partial y_{2}}- \\
{\left[k_{1}\left(k_{1}+1\right)+k_{2}\left(k_{2}-1\right)\right] \tilde{C}=0 }
\end{aligned}
$$

where we denote $\tilde{C}_{\kappa}(Y)$ as $\tilde{C}$.

Let us replace $u=y_{1}+y_{2}$ and $v=y_{1} y_{2}$ in (2), then we find

$$
\left(u^{2}-2 v\right) \frac{\partial^{2} \tilde{C}}{\partial u^{2}}+2 v^{2} \frac{\partial^{2} \tilde{C}}{\partial v^{2}}+2 u v \frac{\partial^{2} \tilde{C}}{\partial u \partial v}+2 u \frac{\partial \tilde{C}}{\partial u}+2 v \frac{\partial \tilde{C}}{\partial v}-\left[k_{1}\left(k_{1}+1\right)+k_{2}\left(k_{2}-1\right)\right] \tilde{C}=0 .
$$

Substituting $z=\frac{u}{2 \sqrt{v}}$ and $t=\sqrt{v}$ we obtain

$$
\left(1-z^{2}\right) \frac{\partial^{2} \tilde{C}}{\partial z^{2}}-t^{2} \frac{\partial^{2} \tilde{C}}{\partial t^{2}}-3 z \frac{\partial \tilde{C}}{\partial z}-t \frac{\partial \tilde{C}}{\partial t}+2\left[k_{1}\left(k_{1}+1\right)+k_{2}\left(k_{2}-1\right)\right] \tilde{C}=0
$$


It is easy to see that the last equation is homogeneous in $t$. Thus by taking

$$
\tilde{C}=t^{k} f(z),
$$

the next ordinary differential equation results

$$
\left(1-z^{2}\right) \frac{d^{2} f}{d z^{2}}-3 z \frac{d f}{d z}+\left[\left(k_{1}-k_{2}\right)\left(k_{1}-k_{2}+2\right)\right] f=0 .
$$

Taking $w=(1-z) / 2$ as the independent variable, the differential equation becomes

$$
w(1-w) \frac{d^{2} f}{d w^{2}}+\frac{3}{2}(1-2 w) \frac{d f}{d w}+\rho(\rho+2) f=0,
$$

with $\rho=k_{1}-k_{2}$, a non negative integer, according to the definition of the partition $\kappa$.

Comparing with the general hypergeometric equation

$$
w(1-w) \frac{d^{2} f}{d w^{2}}+[c-(a+b+1) w] \frac{d f}{d w}-a b f=0,
$$

we see that the complex zonal polynomials are involved in the solution of an hypergeometric differential equation of parameters $a=-\rho, b=\rho+2$ and $c=3 / 2$.

Following Erdélyi et al. (1981), we know that a solution of (4) which is regular at $w=0$ is given by

$$
f(w)=\sum_{n=0}^{\infty} \frac{(a)_{n}(b)_{n}}{(c)_{n} n !} w^{n}={ }_{2} F_{1}(a, b ; c ; w),
$$

where ${ }_{2} F_{1}(a, b ; c ; w)$ is the classical hypergeometric function, which we will denote in front by $F(a, b ; c ; w)$.

Thus a solution of (3) is

$$
f(z)=F\left(-\rho, \rho+2 ; \frac{3}{2} ; \frac{1-z}{2}\right),
$$

Let us refined the above solution by applying properties of the hypergeometric functions. From Erdélyi et al. (1981, Section 2.11, p.111), equation (2), we see that

$$
F\left(2 d, 2 e ; d+e+\frac{1}{2} ; t\right)=F\left(d, e ; d+e+\frac{1}{2} ; 4 t(1-t)\right),
$$

then

$$
\begin{aligned}
f(z) & =F\left(-\rho, \rho+2 ; \frac{3}{2} ; \frac{1-z}{2}\right) \\
& =F\left(-\frac{\rho}{2}, \frac{\rho}{2}+1 ; \frac{3}{2} ; 1-z^{2}\right) .
\end{aligned}
$$

By Erdélyi et al. (1981, Section 2.10, p.108), equation (1),

$$
\begin{aligned}
F(a, b ; c ; t)=A_{1} F(a, b ; a+b-c & +1 ; 1-t) \\
& +A_{2}(1-t)^{c-a-b} F(c-a, c-b ; c-a-b+1 ; 1-t),
\end{aligned}
$$


where

$$
A_{1}=\frac{\Gamma(c) \Gamma(c-a-b)}{\Gamma(c-a) \Gamma(c-b)} \quad \text { and } \quad A_{2}=\frac{\Gamma(c) \Gamma(a+b-c)}{\Gamma(a) \Gamma(b)} .
$$

Then (5) can be written as follows

$$
\begin{aligned}
F\left(-\frac{\rho}{2}, \frac{\rho}{2}+1 ; \frac{3}{2} ; 1-z^{2}\right)=A_{1} F\left(-\frac{\rho}{2}, \frac{\rho}{2}+1 ; \frac{1}{2} ;\right. & \left.z^{2}\right) \\
& +A_{2} z F\left(\frac{3}{2}+\frac{\rho}{2}, \frac{1}{2}-\frac{\rho}{2} ; \frac{3}{2} ; z^{2}\right)
\end{aligned}
$$

where

$$
A_{1}=\frac{\Gamma\left(\frac{3}{2}\right) \Gamma\left(\frac{1}{2}\right)}{\Gamma\left(\frac{3}{2}+\frac{\rho}{2}\right) \Gamma\left(\frac{1}{2}-\frac{\rho}{2}\right)} \quad \text { and } \quad A_{2}=\frac{\Gamma\left(\frac{3}{2}\right) \Gamma\left(-\frac{1}{2}\right)}{\Gamma\left(-\frac{\rho}{2}\right) \Gamma\left(\frac{\rho}{2}+1\right)} .
$$

If $\rho=k_{1}-k_{2}=2 n, n=0,1,2, \ldots$, and using the fact that $\Gamma\left(\frac{1}{2}+z\right) \Gamma\left(\frac{1}{2}-z\right)=\pi \sec (z \pi)$ and $\Gamma(z) \Gamma(1-z)=\pi \csc (z \pi)$ which implies $A_{1}=\frac{(-1)^{n}}{2 n+1}$ and $A_{2}=0$, respectively, then the even complex zonal polynomials of second order are given by

$$
\tilde{C}_{\left(k_{1}, k_{2}\right)}(Y) / \tilde{C}_{\left(k_{1}, k_{2}\right)}\left(I_{2}\right)=\left(y_{1} y_{2}\right)^{\frac{k}{2}} \frac{(-1)^{n}}{2 n+1} F\left(-n, n+1 ; \frac{1}{2} ; \frac{\left(y_{1}+y_{2}\right)^{2}}{4 y_{1} y_{2}}\right) .
$$

If $\rho=k_{1}-k_{2}=2 n+1, n=0,1,2, \ldots$ and using the same properties for the simplification of A's then we find the odd complex zonal polynomials of second order

$$
\tilde{C}_{\left(k_{1}, k_{2}\right)}(Y) / \tilde{C}_{\left(k_{1}, k_{2}\right)}\left(I_{2}\right)=\left(y_{1} y_{2}\right)^{\frac{k}{2}}(-1)^{n} \frac{y_{1}+y_{2}}{2 \sqrt{y_{1} y_{2}}} F\left(n+2,-n ; \frac{3}{2} ; \frac{\left(y_{1}+y_{2}\right)^{2}}{4 y_{1} y_{2}}\right),
$$

where $z^{2}<1$ and

$$
\tilde{C}_{\left(k_{1}, k_{2}\right)}\left(I_{2}\right)=k ! \frac{\left(k_{1}-k_{2}+1\right)^{2}}{\left(k_{1}+1\right) !\left(k_{2}\right) !}
$$

see Khatri (1970).

These formulae agree with the expressions found for the complex zonal polynomials in Farrell (1980) until the 5th degree.

\section{Acknowledgments}

This research work was partially supported by CONACYT-México, research grant no. 45974-F.

\section{References}

Díaz-García, J.A.; Caro, F.J. (2006a) "An alternative approach for deriving the LaplaceBeltrami operator for the zonal polynomials of positive semidefinite and definite matrix argument", Far East Journal of Mathematical Sciences (FJMS) 22(3): 273-290. 
Díaz-García, J.A.; Caro, F.J. (2006b) "Derivation of the Laplace-Beltrami operator for the zonal polynomials of positive definite Hermitian matrix argument". Submitted.

Erdélyi, A.; Magnus, W.; Oberhettinger, F.; Tricomi, F.; Bertin, D.; Fulks, W.; Harvey, A.; Thomsen, D.; Weber, M.; Whitney E. (1981) Higher Trascendeltal Functions 1. Robert E. Krieger Publishing Company, Malabar.

Farrell R.H. (1980) "Calculation of complex zonal polynomials", in: P.R. Kishnaiah (Ed.) Multivariate Analysis V, North Holland: 301-320.

James, A. T. (1968) "Calculation of zonal polynomial coefficients by use of the LaplaceBeltrami operator", Ann. Math. Statist. 39: 1711-1718.

Khatri, C. G. (1907) "On the moments of traces of two matrices in three situations for complex multivariate normal populations", Sankhyā, A 32: 65-80. 